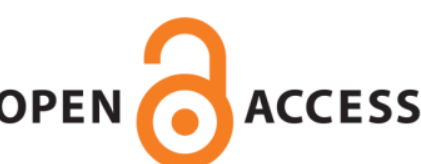

UWS Academic Portal

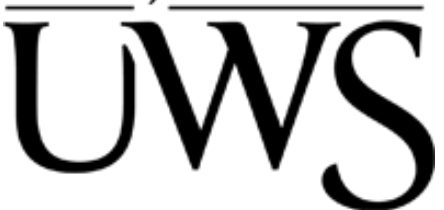

\title{
Intracell interference characterization and cluster interference for D2D communication
}

Mustafa, Hafiz Attaul; Ekti, Ali Riza; Shakir, Muhammad Zeeshan; Imran, Muhammad Ali;

Tafazolli, Rahim

Published in:

IEEE Transactions on Vehicular Technology

DOI:

10.1109/TVT.2018.2850820

Published: 26/06/2018

Document Version

Peer reviewed version

Link to publication on the UWS Academic Portal

Citation for published version (APA):

Mustafa, H. A., Ekti, A. R., Shakir, M. Z., Imran, M. A., \& Tafazolli, R. (2018). Intracell interference

characterization and cluster interference for D2D communication. IEEE Transactions on Vehicular Technology, 67(9), 8536-8548. https://doi.org/10.1109/TVT.2018.2850820

\section{General rights}

Copyright and moral rights for the publications made accessible in the UWS Academic Portal are retained by the authors and/or other copyright owners and it is a condition of accessing publications that users recognise and abide by the legal requirements associated with these rights.

Take down policy

If you believe that this document breaches copyright please contact pure@uws.ac.uk providing details, and we will remove access to the work immediately and investigate your claim. 
(C) 2018 IEEE. Personal use of this material is permitted. Permission from IEEE must be obtained for all other uses, in any current or future media, including reprinting/republishing this material for advertising or promotional purposes, creating new collective works, for resale or redistribution to servers or lists, or reuse of any copyrighted component of this work in other works. 


\title{
Intracell Interference Characterization and Cluster Interference for D2D Communication
}

\author{
Hafiz Attaul Mustafa ${ }^{1}$, Ali Riza Ekti ${ }^{2}$, Muhammad Zeeshan Shakir ${ }^{3}$, \\ Muhammad Ali Imran ${ }^{4}$, and Rahim Tafazolli ${ }^{1}$ \\ ${ }^{1}$ Institute for Communication Systems, University of Surrey, Guildford, UK. \\ \{h.mustafa, r.tafazolli\}esurrey.ac.uk \\ ${ }^{2}$ Department of Electrical and Electronics Engineering, Balikesir University, Turkey. \\ arekti@balikesir.edu.tr \\ ${ }^{3}$ School of Engineering and Computing, University of the West of Scotland, Paisley, UK. \\ muhammad.shakireuws.ac.uk \\ ${ }^{4}$ School of Engineering, University of Glasgow, Glasgow, UK. \\ Muhammad.Imran@glasgow.ac.uk
}

\begin{abstract}
The homogeneous spatial Poisson point process (SPPP) is widely used for spatial modeling of mobile terminals (MTs). This process is characterized by a homogeneous distribution, complete spatial independence, and constant intensity measure. However, it is intuitive to understand that the locations of MTs are neither homogeneous, due to inhomogeneous terrain, nor independent, due to homophilic relations. Moreover, the intensity is not constant, due to mobility. Therefore, assuming an SPPP for spatial modeling is too simplistic, especially for modeling realistic emerging device-centric frameworks such as device-to-device (D2D) communication. In this paper, assuming inhomogeneity, positive spatial correlation, and random intensity measure, we propose a doubly stochastic Poisson process, a generalization of the homogeneous SPPP, to model D2D communication. To this end, we assume a permanental Cox process (PCP) and propose a novel Euler-Characteristic-based approach to approximate the nearest-neighbor distribution function. We also propose a threshold and spatial distances from an excursion set of a chi-square random field as interference control parameters for different cluster sizes. The spatial distance of the clusters is incorporated into a Laplace functional of a PCP to analyze the average coverage probability of a cellular user. A closedform approximation of the spatial summary statistics is in good agreement with empirical results, and its comparison with an SPPP authenticates the correlation modeling of D2D nodes.
\end{abstract}

Index Terms-Intracell interference, D2D communication, spatial correlation, permanental Cox process, random field, Euler Characteristic, nearest-neighbor distribution function.

\section{INTRODUCTION}

A homogeneous Poisson point process (PPP) is characterized by the remarkable property of complete spatial randomness. This property is useful when the underlying points are completely uncorrelated with each other and, subsequently, are distributed homogeneously. For example, call arrivals in a cellular network can be precisely modeled by a temporal PPP if we ignore traffic inhomogeneity between day and night periods. The spatial version of a PPP (SPPP) is extensively used to model the position of base stations (BSs) and mobile terminals (MTs) [1]-[3]. However, BSs/MTs are neither uncorrelated nor distributed homogeneously. Moreover, due to spatial variations in traffic, the intensity measure of the point process cannot be considered constant. The inhomogeneity and spatial correlation is usually governed by several dominant factors, such as strategical deployment of BSs, homophilic relations between MTs, emergence of mobile social networks, and the existence of hot-spots. As a result, a homogeneous PPP is too simplistic to model the temporal/spatial topologies of network entities.

\section{A. Motivation}

In present and future heterogeneous networks, mobile social networks are evolving rapidly. According to the Statistics Portal, the social network users are expected to increase from 1.91 billion in 2014 to 2.67 billion in 2018 [4]. For such cellular networks, ignoring the correlation (temporal, spatial, or both) between nodes of BS and MT clusters for stochastic modeling is questionable. Another factor is the inhomogeneous deployment of BSs and MTs due to inhomogeneous terrain in real cellular networks. Moreover, the intensity of BSs/MTs ranges from constant values (fixed deployment of BSs) to random values (mobility and hand-overs of MTs). For realistic spatial modeling, we consider two key phenomena in this paper, i.e., inhomogeneity and positive correlation between MTs in the coverage area. As a matter of fact, many applications, e.g., proximity services, caching, content sharing, and D2D/M2M, depend on this very correlation. For example, in content-sharing applications, two MTs having common interests, can exploit spatial correlation in the form of physical nearness. In order to highlight the motivation of this research, we present some key questions that need to be addressed by candidate spatial point process:

1) Is the probability of occurrence of MTs the same at any location in the coverage area?

2) From the perspective of any social relation or physical bond, are MTs completely independent from each other?

3) Does the existence of homophilic relation disturb the spatial homogeneity?

4) Is the user intensity in the coverage area constant or does it change randomly due to hand-overs?

In spatial modeling, the widely used homogeneous SPPP cannot precisely model cellular networks since it cannot 
capture negative correlation (repulsion), in the case of BSs, or positive correlation (attraction), in the case of MTs. Moreover, it cannot address the above questions. By generalizing the SPPP to a process where the distribution is inhomogeneous, points have spatial correlation, and user intensity is random, we can realize an accurate spatial modeling of MTs. The relevant processes, which capture negative and positive spatial correlations, are called fermion and boson [5]. These processes can be modeled, respectively, by a determinantal point process (DPP) and a permanental Cox process (PCP) [6]. The PCP is a doubly stochastic Poisson process, with random intensity measure and positive spatial correlation, governed by a chisquare random field ( $\left.\chi_{k}^{2}-\mathrm{RF}\right)$ with $k$ degrees of freedom (DoF).

\section{B. Related Work}

The negative correlation between BSs has been modeled using a DPP or Ginibre point process [7]-[9]. However, to our best knowledge, the spatial modeling of MTs is restricted to a homogeneous SPPP in the literature. This paper is the first attempt to model an inhomogeneous distribution of MTs with spatial correlation that exists due to any homophilic relation. Thus, we extend our SPPP approach [10], [11] to a PCP model with random intensity measure and inhomogeneous distribution to characterize interference in an underlay D2D network. To validate simulated realizations, we used an $n$ thorder product density of the PCP to derive Ripley's $K$ and variance-stabilized $L$ functions. These functions are compared with a benchmark SPPP process to see the deviations. More upper deviation means higher positive correlation between points of the process. The $K$ and $L$ functions of various point processes are available [8], [12], however, no analytic expressions for the PCP exist in the literature. The random intensity measure of a PCP is approximated by topological inference, based on the expected Euler Characteristic (EC) [13]. This approximation is used to derive the nearest-neighbor distribution function, which is introduced into a Laplace functional of the PCP to capture interference due to D2D pairs. The threshold of the excursion set of a $\chi_{k}^{2}-\mathrm{RF}$ and the physical distance between D2D pairs are introduced as interference control parameters to analyze and ensure average coverage probability of a cellular user.

\section{Contributions}

1) Using an $n$ th-order product density of the PCP, we derive the $K$ and $L$ functions for the exponential covariance function.

2) We propose an expected EC-based novel approach to approximate the random intensity measure of a PCP, which is governed by a $\chi_{k}^{2}$-RF with $k$ DoF.

3) Inspired by statistical parameter mapping and random field theory (RFT) approaches ${ }^{1}$ towards functional anal-

\footnotetext{
${ }^{1}$ In standard functional analysis of the brain, two approaches are followed to identify activation regions against the null hypothesis (e.g., z-test, $\chi^{2}$-test, t-test, F-test): Bonferroni correction, and random field theory. The functional analysis of the brain comprises a large number of voxels, i.e., statistical values. In case the statistical values are completely independent, the former approach is best to identify activation regions. However, if spatial correlation exists, the latter approach provides a less conservative analysis and accurate identification of activation regions.
}

ysis of brain imaging [14], we adopt the RFT approach to derive a closed-form approximation (CFA) for an intractable nearest-neighbor distribution function $G$.

4) We introduce the $G$ function into a Laplace functional of the PCP to capture interference, and subsequently derive a CFA for the average coverage probability of a cellular user in a D2D underlay network.

5) We propose a threshold $u$ of the excursion set of a $\chi_{k}^{2}$-RF and spatial distance $r$ (physical distance between D2D pairs) as interference control parameters to characterize intracell interference, and analyze the coverage probability of a cellular user in an underlay D2D network.

\section{Organization}

The rest of the paper is organized as follows: In Section II, we provide the required mathematics of random fields, point processes, excursion sets, and Lipschitz-Killing curvature measures to understand the ideas proposed in this research. In Section III, we present a system model of the cellular network with underlay D2D communication. This is followed by a PCP model for the spatial distribution of potential D2D nodes, and details to generate such a process. To validate the simulated realizations of the PCP, we also derive a CFA of $K$ and $L$ functions in this section. In Section IV, we present the main result of approximating the $G$ function of a PCP. A CFA of the $G$ function is derived based on the expected EC of the excursion set of a $\chi_{k}^{2}$-RF. The CFA and empirical $G$ function are compared with an SPPP process. In Section V, we introduce the $G$ function into a Laplace functional of a PCP to derive a CFA for the average coverage probability of a cellular user. Conclusions are drawn in Section VI.

\section{MAThematical PREliminaries}

1) Random Field: A random field (RF) on the subspace of a stratified manifold $M \subset \mathbb{R}^{k}$, a $k$-dimensional vector space, can be defined as a function $f=f(t)$ whose values are random variables (RVs) for any $t \in M$ [15]. This function is fully characterized by its finite-dimensional (FiDi) distribution, i.e.,

$$
F_{t_{1}, \ldots t_{k}}\left(x_{1}, \ldots, x_{k}\right)=p\left[f\left(t_{1}\right) \leq x_{1}, \ldots, f\left(t_{k}\right) \leq x_{k}\right] .
$$

If (1) is multivariate Gaussian, $f$ is known as a Gaussian RF (GRF). However, in the real world, not all RFs are Gaussian. Non-Gaussian fields form a very broad class and are not well defined. Here, we consider RFs of the form $h(t)=H\left[f_{1}(t), \ldots, f_{k}(t)\right]$ where $H(\cdot)$ is any functional transformation and $f_{k}(t)$ is the $k$ th RF on subspace $t$. Such types of GRFs are classified as non-Gaussian or Gaussian-related $\mathrm{RFs}^{2}$ [13], [16]. Assuming $f_{1}(t), \ldots, f_{k}(t)$ as independent, identically distributed (i.i.d.), homogeneous, real-valued GRFs

${ }^{2}$ For independent, identically distributed, homogeneous, real-valued GRFs, i.e., $X_{1}(t), \ldots, X_{n}(t), Y_{1}(t), \ldots, Y_{m}(t)$, some of the famous non-Gaussian or Gaussian-related RFs can be defined as:

- $\chi_{n}^{2}$-field $=\sum_{i=1}^{n} X_{\mathrm{i}}^{2}(t) ; \quad H$ is a square function.

- $F$-field $=\frac{\sum_{i=1}^{n} X_{\mathrm{i}}^{2}(t) / n}{\sum_{i=1}^{m} Y_{\mathrm{i}}^{2}(t) / m} ; \quad H$ is a scaled ratio.

- $t$-field $=\frac{X_{i=1}(t)}{\left[\sum_{i=1}^{m} Y_{\mathrm{i}}^{2}(t) / m\right]^{\frac{1}{2}}} ; \quad H$ is a normalization function. 
with zero-mean and unit-variance, the transformation $H(\cdot)$ as the square function defines a well known class of nonGaussian RFs, i.e., $\chi_{k}^{2}$-RF [16]:

$$
h(t)=\sum_{m=1}^{k} f_{m}^{2}(t) .
$$

The marginal distribution of (2), for each $t \in M$, is $\chi^{2}$ with $k$ DoF.

2) Permanental Cox Process: We define a spatial point process $\Phi$ in terms of an $n$ th-order product density $\varrho^{(n)}$. Process $\Phi$ is a random subset $X$ of an underlying locally compact topological/parameter space $S$, a subspace of a stratified manifold $M \subset \mathbb{R}^{k}$. A process $\Phi$ is said to be a PCP process if $X$ is Poisson with a random intensity measure defined as [17]:

$$
\begin{aligned}
\Lambda(B) \stackrel{a . s .}{=} & \int_{B} \lambda(s) d s, \\
& =\int_{B}\left[Y_{1}^{2}(s)+\cdots+Y_{k}^{2}(s)\right] d s, \\
& =\int_{B} \chi_{k}^{2} d s,
\end{aligned}
$$

where $B \subseteq S$ is a Borel set, $\lambda(s)$ is a random intensity function, and $Y_{(\cdot)}(s)$ are $k$ independent GRFs.

3) Excursion Set: The excursion set, where $f(t)$ is above level $u \in \mathbb{R}$, of a $k$-dimensional $\mathrm{RF}$ on $M$ is given as [18], [19]:

$$
A_{u}(f, M) \triangleq[t \in M: f(t) \geq u] \equiv f^{-1}\{[u,+\infty)\} .
$$

The excursion set of a real-valued non-Gaussian RF can be defined by applying the function composition $h=(H \circ f)$ on $M$. This set is equivalent to the excursion set of a vectorvalued Gaussian function $f$ in $H^{-1}[u,+\infty)$, which, under appropriate assumptions on $H$, is a manifold with a piecewise smooth boundary given by [13]:

$$
\begin{aligned}
A_{u}(h, M) & =A_{u}[(H \circ f), M], \\
& =[t \in M:(H \circ f)(t) \geq u], \\
& =\left\{t \in M: f(t) \in H^{-1}[u,+\infty)\right\}, \\
& =M \cap f^{-1}\left\{H^{-1}[u,+\infty)\right\} .
\end{aligned}
$$

Since, $H^{-1}[u,+\infty)$ is a specific stratified manifold in $\mathbb{R}^{k}$, we can generalize it to $D \subset \mathbb{R}^{k}$ in (4) as:

$$
A_{u}(h, M)=M \cap f^{-1}(D) .
$$

4) Lipschitz-Killing Curvature Measures: The Euler Characteristic $\mathcal{X}$ is a fundamental additive functional that counts the topological components of $M$. In order to consider the boundary, curvature, surface area, and volume of $M$, positioninvariant and rotation-invariant generalized functionals are considered that are known as Lipschitz-Killing curvature measures. They are also known as geometric identifiers that capture the intrinsic volume of $M$. For example, in the case of $M \subset \mathbb{R}^{2}, \mathcal{L}_{0} \equiv \mathcal{X}, \mathcal{L}_{1}$, and $\mathcal{L}_{2}$ give the EC, boundary length,

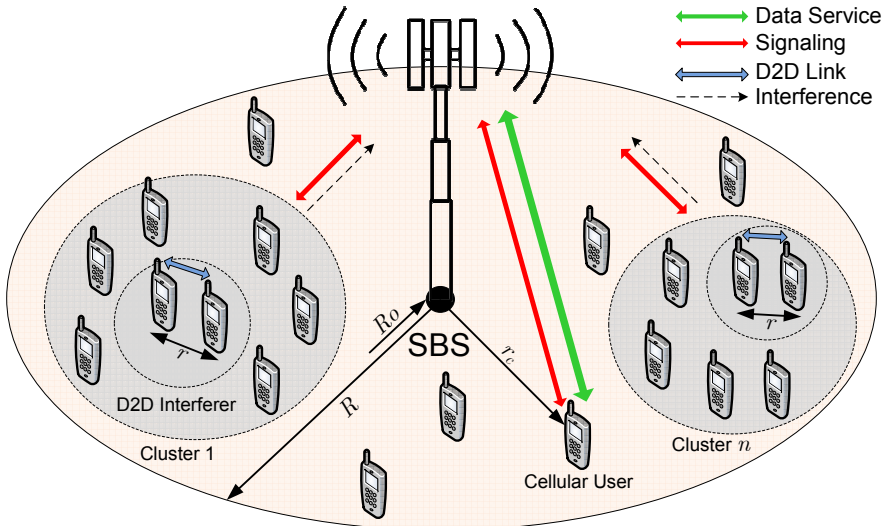

Fig. 1: System model for PCP-deployed MTs.

and area of the manifold $M$, respectively. The LipschitzKilling curvature measures $\mathcal{L}_{j}$ on $B_{R}^{N}$, the $N$-dimensional ball of radius $R$, are given as [13, Section 6.3]:

$$
\mathcal{L}_{j}\left(B_{R}^{N}\right)=\left(\begin{array}{c}
N \\
j
\end{array}\right) R^{j} \frac{w_{N}}{w_{N-j}},
$$

where $j$ extends to the dimension of $M$ (i.e., $j=|M|$ ) and $\omega_{n}$ is the volume of the unit ball in $\mathbb{R}^{n}$.

\section{System MODEL}

In this section, we present a cellular network model, a PCP model, a method of process generation, and validation procedure using $K$ and $L$ functions.

\section{A. Cellular Network Model}

The cellular network comprises a small-cell BS (SBS) and MTs, as shown in Fig. 1. In a multi-cell scenario, SBSs can be modeled by a DPP or Ginibre process that characterizes repulsion due to strategic deployment, instead of random deployment. However, we focus on the spatial distribution of MTs in a single-cell scenario. We consider an orthogonal frequency-division multiple access (OFDMA)based cellular network. In this case, we want to analyze the maximum frequency reuse and the effect of interference due to D2D communication. In order to avoid coverage holes, the SBS should provide homogeneous coverage for cellular users. Accordingly, we consider one MT as a cellular user that is uniformly distributed. All other MTs are potential D2D users, distributed according to the PCP process. The existence of MTs, in the form of clusters, are due to the spatial correlation of the PCP process. This spatial correlation can be governed by any type of homophilic relation. Such clusters of MTs can be realized as D2D clusters for proximity services, as shown in Fig. 1.

The uplink resources of the cellular user are shared by potential D2D users. The time-division duplex (TDD) mode is assumed between D2D nodes to capture the effect of interference generated by both nodes. In the case of frequencydivision duplex (FDD), the interference at any time instant will simply be half that of the TDD mode. The data and signaling are provided by the serving SBS to the cellular 
user, whereas only signaling is assumed for potential D2D nodes. For the average coverage probability of a cellular user, the interference is generated by all successful D2D pairs. We consider interference at the serving SBS from successful D2D nodes in neighboring SBSs negligible due to the low transmit power.

The cellular and potential D2D users are distributed in the coverage area bounded between the SBS radius $R$ and the protection region $R_{0} \approx 0$. The distance between the SBS and a cellular user is $r_{\mathrm{c}}$, which is used to calculate path loss at the SBS. Every successful D2D pair has a distance of $r$ between nodes. The channel model assumes distancedependent path loss and Rayleigh fading. The simple singular path loss model $\left(r_{\mathrm{c}}{ }^{-\alpha}\right)$ is assumed, where the protection region $R_{0}$ ensures convergence of the model by avoiding nodes at the origin. The received power at the SBS follows an exponential distribution. The distance $r_{\mathrm{c}}$ follows the density function $^{3}$ as in [20, Theorem 5, Algorithm 3, pp. 17-18]:

$$
f\left(r_{\mathrm{c}}\right)=\frac{2 r_{\mathrm{c}}}{R^{2}}, f(\theta)=\frac{1}{2 \pi},
$$

where $R_{0} \leq r_{\mathrm{c}} \leq R$ and $0 \leq \theta \leq 2 \pi$.

\section{B. PCP Model}

Definition 1. The $n$ th-order product density $\varrho^{(n)}$ of a Cox process is [21]:

$$
\varrho^{(n)}\left(s_{1}, \ldots, s_{n}\right)=\mathrm{E} \prod_{i=1}^{n} \Lambda\left(s_{\mathrm{i}}\right),
$$

where $\Lambda(\cdot)$ is a random intensity measure ${ }^{4}$. In order to model a spatially correlated process for potential D2D nodes, we consider a PCP with the following intensity measure:

$$
\Lambda\left(s_{\mathrm{i}}\right)=Y_{1}^{2}\left(s_{\mathrm{i}}\right)+\cdots+Y_{k}^{2}\left(s_{\mathrm{i}}\right),
$$

where $Y_{(\cdot)}(\cdot)$ are $k$ zero-mean unit-variance independent realvalued stationary GRFs and $Y_{(\cdot)}^{2}(\cdot)=\chi^{2}(\cdot)$ with one DoF.

The sum of independent chi-square distributions results in a $\chi_{k}^{2}$-RF with $k$ DoF [22] that reduces the intensity measure of the PCP to:

$$
\Lambda\left(s_{\mathrm{i}}\right)=\chi_{k}^{2}\left(s_{\mathrm{i}}\right) .
$$

Since the distribution of potential D2D nodes is translationinvariant and motion-invariant, we can assume a stationary PCP, and hence borrow the following definition from [17]:

Definition 2. A PCP is stationary if and only if the underlying GRF is stationary.

\footnotetext{
${ }^{3}$ This density function requires normalization over $r_{\mathrm{c}} \in[0, \infty)$ to become a probability density function. In our analysis, the area of interest is the coverage area, i.e., a circle of radius $R\left(0 \leq r_{\mathrm{c}} \leq R\right)$. For such an area, the density function is already normalized since $\int_{0}^{R} \frac{2 r_{\mathrm{c}}}{R^{2}} d r_{\mathrm{c}}=1$.

${ }^{4}$ In measure theory, a measure is a systematic number assigned to quantify the measurement of interest, e.g., count, length, area, volume, probability, intensity etc. In a point process, the most important measure is the random intensity measure, which captures the random intensity of points in the area of interest.
}

The stationarity of a GRF is ensured by the underlying covariance function. In order to generate smooth GRFs, we consider a squared exponential covariance function [23]:

$$
\operatorname{Cov}\left(s_{1}, s_{2}\right)=e^{-\frac{\|s\|^{2}}{2 l^{2}}}
$$

where $\|s\|=\left\|s_{1}-s_{2}\right\|=\sqrt{\left(s_{1_{x}}-s_{2_{x}}\right)^{2}+\left(s_{1_{y}}-s_{2_{y}}\right)^{2}}$ is the Euclidean distance between $s_{1}$ and $s_{2}$, and $l$ is the characteristic length-scale. The resulting covariance matrix of the PCP is defined as:

$$
\begin{aligned}
& {[\mathrm{C}]\left(s_{1}, \ldots, s_{n}\right)} \\
& =\left[\begin{array}{cccc}
1 & \operatorname{Cov}\left(s_{1}, s_{2}\right) & \cdots & \operatorname{Cov}\left(s_{1}, s_{n}\right) \\
\operatorname{Cov}\left(s_{2}, s_{1}\right) & 1 & \cdots & \operatorname{Cov}\left(s_{2}, s_{n}\right) \\
\vdots & & \ddots & \vdots \\
\operatorname{Cov}\left(s_{n}, s_{1}\right) & \operatorname{Cov}\left(s_{n}, s_{2}\right) & \cdots & 1
\end{array}\right] .
\end{aligned}
$$

Definition 3. The $n$ th-order product density of a Cox process is equal to the weighted permanent ${ }^{5} \operatorname{per}_{\alpha}$ of the covariance matrix:

$$
\varrho^{(n)}\left(s_{1}, \ldots, s_{n}\right)=\operatorname{per}_{\alpha}[\mathbf{C}]\left(s_{1}, \ldots, s_{n}\right) .
$$

For a proof of the fact that (8) in Definition 1 and (11) in Definition 3 are equivalent, the interested reader is referred to [21, Sec. 2.1.1, pp. 876, Theorem 1, Lemma 1].

Equation (11) is a useful representation to derive the summary statistics of the PCP process. The summary statistics, in this research, depend on the first- and second-order product density. By the fact that the underlying GRF of a Cox process is stationary, the argument $\left(s_{1} ; s_{2}\right)$ in all summary statistics can be replaced by $s$. The value of $\alpha$ characterizes the underlying point process. For example, a PCP process, which captures positive correlation, is effectively a boson (or photon) process with $\alpha=1$ [26], resulting in $k=2 \mathrm{DoF}$ for the underlying GRFs.

\section{Process Generation}

The random intensity measure of a PCP is governed by a $\chi_{k}^{2}$-RF that is non-Gaussian or Gaussian-related. Since the component field is a GRF, it can be generated by drawing real-valued multivariate normal random vectors and mapping these to the underlying grid. It can also be generated via the circulant embedding method [27]. We followed the former approach to generate RFs and subsequently the PCP process. The $\chi_{k}^{2}$-RF of the PCP comprises a large number of $\chi^{2} \mathrm{RVs}$, which are mapped to each grid point $s \in S$. Due to the

${ }^{5}$ The weighted permanent $\operatorname{per}_{\alpha}$ of covariance matrix $[\mathrm{C}]\left(s_{1}, \ldots, s_{n}\right)$ is defined as:

$$
\operatorname{per}_{\alpha}[\mathrm{C}]\left(s_{1}, \ldots, s_{n}\right)=\sum_{\sigma \in \mathrm{G}_{n}} \alpha^{r} \prod_{i=1}^{n}\left[s_{\mathrm{i}}, s_{\sigma(i)}\right],
$$

where permutation is carried out on every element $\sigma$ of a symmetric group $\mathrm{G}_{n}$. The resulting polynomial is $\alpha$ weighted by a coefficient of degree $r$ over permutations of exactly $r$ cycles [21]. The usual permanent of a matrix has been proved to be in the sharp polynomial complexity class, i.e., a \#P-complete problem due to a theorem of Valiant [24]. The weighted permanent is not known to be \#P-complete, however, it is equally difficult as the \#P-complete problem. For the solution to such problems, approximation approaches using Markov chain Monte Carlo algorithms exist in the literature [25]. 


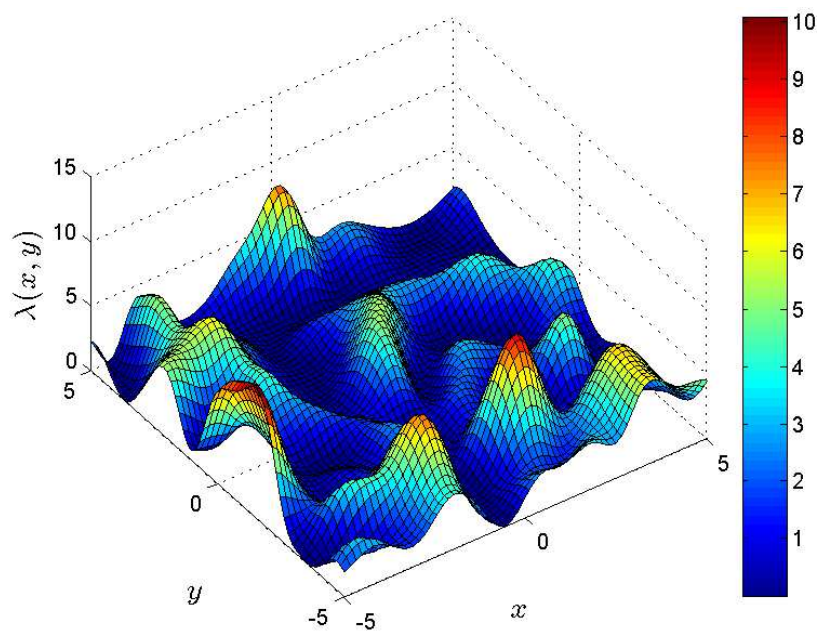

(a)

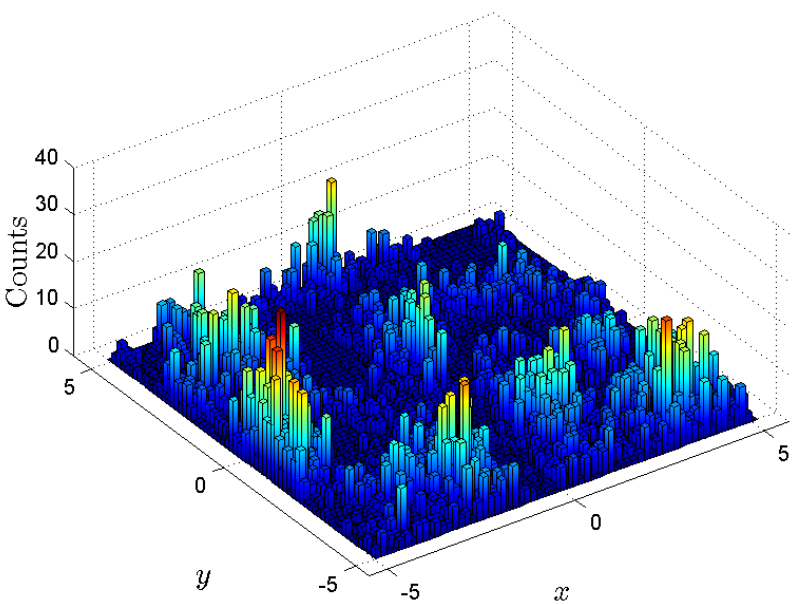

(b)

Fig. 2: Random intensity function of a PCP process with two DoF and its sampled histogram. (a) Intensity function resembles bell-like blobs of a GRF. (b) Sampled realization shows high and low intensity regions.

smooth underlying covariance structure, each RV results in a smooth sample path. The blobs and holes show spatial covariance between the $\chi^{2}$ RVs. The overall shape of the intensity measure of the PCP is similar to the symmetric belllike blobs of a GRF, however, the loss of symmetry in this case is due to the finite DoF of the $\chi_{k}^{2}-\mathrm{RF}$. For infinite DoF, due to the central limit theorem, the intensity measure of the PCP also resembles the symmetric bell-like blobs of a GRF.

The lattice representation of a $\chi_{k}^{2}-\mathrm{RF}$ with two DoF is shown in Fig. 2(a). In this figure, we can see a number of blobs and holes that, respectively, show high and low intensity areas. The high-intensity areas $(7 \rightarrow 10$ on color bar) capture strong spatial correlation between the points, and results in group clustering, whereas low-intensity areas $(1 \rightarrow 3$ on color bar) form holes due to the nonexistence of any homophilic relation. The Markov chain Monte-Carlo-based MetropolisHasting (MH) sampler ${ }^{6}$ is used to sample PCP points under a $\chi_{k}^{2}$-RF, shown in Fig. 2(b) as an inhomogeneous and clustered distribution of points.

\section{Summary Statistics: $K$ and L Functions}

In order to realize D2D communication in real cellular networks, the foremost step is to find dependence or spatial correlation between mobile users. In the context of D2D modeling using a point process, this comes under spatial analysis methods that are used to find dependence or correlation between points. In the literature, two important functions are reported as Ripley's $K$ function and the variance-stabilized $L$ function, which are, respectively, defined as [29]:

$$
K(r)=\int_{0}^{r} g(s) 2 \pi s d s,
$$

${ }^{6}$ The MH sampler is used to sample RVs from multidimensional spaces. The states of the underlying Markov chain can be updated in two different ways, 1) block-wise, and 2) component-wise. The first approach updates all state variables simultaneously, whereas the second approach iterates with component-wise updates [28].

$$
L(r)=\sqrt{\frac{K(r)}{\pi}},
$$

where $r$ is the distance and $g(s)$ is the pair correlation function. Using (11), the first-order and second-order product densities can be, respectively, derived as [30]:

$$
\varrho=\alpha, \quad \varrho^{(2)}(s)=\alpha\left[1+\operatorname{Cov}^{2}(s)\right],
$$

where $\operatorname{Cov}(s)$ is a squared exponential covariance function. Since $g(s)=\varrho^{(2)}(s) / \varrho^{2}$, the pair correlation function of the PCP for $\alpha=1$ is given by:

$$
g(s)=1+\operatorname{Cov}^{2}(s) .
$$

The corresponding $K$ and $L$ functions can be derived as:

$$
\begin{aligned}
K(r) & =\pi r^{2}+\pi l^{2}\left[1-e^{-\left(\frac{r}{l}\right)^{2}}\right], \\
L(r) & =\sqrt{r^{2}+l^{2}\left[1-e^{-\left(\frac{r}{l}\right)^{2}}\right]} .
\end{aligned}
$$

The SPPP is a special case of a PCP with $g(s)=1$, $K(r)=\pi r^{2}$, and $L(r)=r$. If we assume complete spatial independence, the covariance between $s_{1}$ and $s_{2}$ vanishes and the product densities from (12) reduce to:

$$
\varrho=\alpha, \quad \varrho^{(2)}(s)=\alpha[1+0] .
$$

The corresponding pair correlation function (for $k=2$, i.e., $\alpha=1$ ) is 1 . The $K$ and $L$ functions for an SPPP can be evaluated as $K(r)=\pi r^{2}$ and $L(r)=r$, respectively. The analytic expression of the $K$ and $L$ functions and empirical estimates are shown in Fig. 3. As an illustration, the plot is shown for a value of $l=50$. This parameter captures the length-scale of the underlying sample path. In a modeling problem, it can be used to incorporate the level of covariance between points of the process. We can see that the estimates of the $K$ and $L$ functions match the CFAs. In this figure, the SPPP serves as a benchmark with zero correlation between points. The positive spatial correlation of the PCP can be verified by the upper drift of the $K$ and $L$ functions. In case 


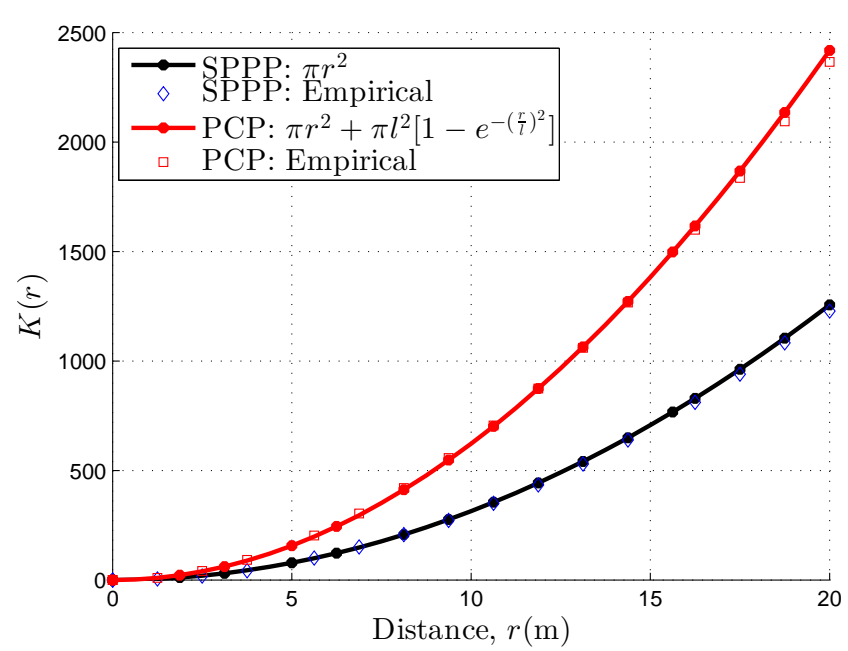

(a)

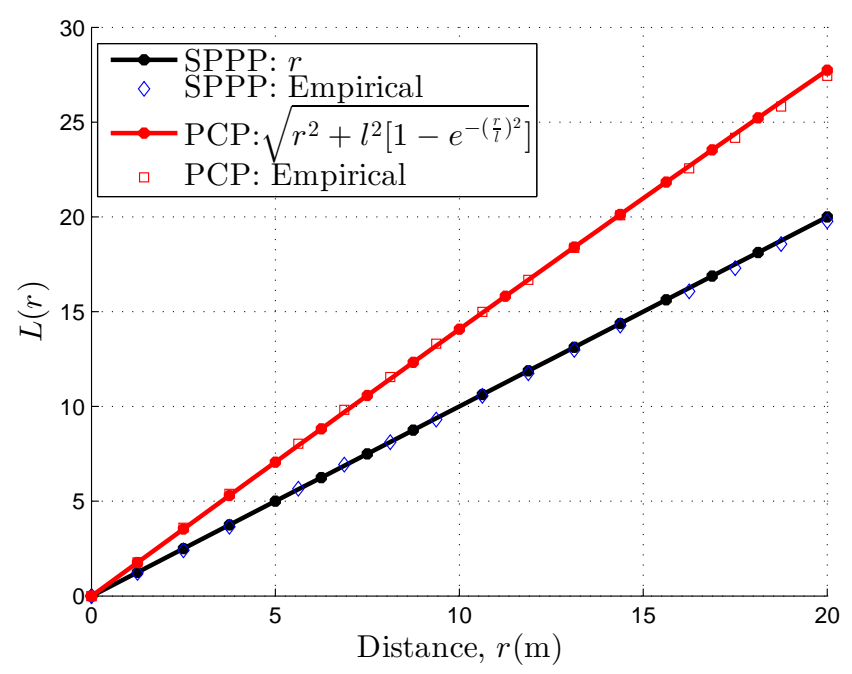

(b)

Fig. 3: CFA and empirical approximations for summary statistics of SPPP and PCP processes for $l=50$. (a) $K$ function. (b) $L$ function.

of negative spatial correlation, the $K$ and $L$ functions should lie below the SPPP curves [7]-[9].

\section{NeAREST-Neighbor Distribution Function}

In this section, we approximate the $G$ function using topological inference based on the expected EC and Poisson clumping heuristic [31]. To this end, we first approximate $\Lambda(\cdot)$ of the PCP as the expected intensity measure $\psi_{0}$ using the EC approach. This is followed by considering $\psi_{0}$ in the Poisson clumping heuristic to approximate the $G$ function. Numerical validation and comparison with an SPPP will also be presented.

Definition 4. The nearest-neighbor distribution function of a general point process is given as:

$$
\begin{aligned}
G(r) & =1-\mathbb{E}\left[e^{-\Lambda\left(B_{r}^{N}\right)}\right], \\
& =1-\mathbb{E}\left[e^{-\int_{B_{r}^{N}} \lambda(s) d s}\right],
\end{aligned}
$$

where $\Lambda\left(B_{r}^{N}\right)$ is the intensity measure over $B_{r}^{N}$, which is an $N$-dimensional closed ball of radius $r$ at an arbitrary position, and $\lambda(\cdot)$ is the intensity function of a point process .

In the case of an SPPP, $\lambda$ is constant, and hence (13) can be simplified to:

$$
G(r)=1-e^{-\lambda \pi r^{2}}
$$

Considering $\Lambda(\cdot)$ from (9), the nearest-neighbor distribution function of the PCP is given by:

$$
\begin{aligned}
G(r) & =1-\mathbb{E}\left(e^{-\int_{B_{r}^{N}} \chi_{k}^{2} d s}\right), \\
& =1-\mathbb{E}\left(e^{-\int_{B_{r}^{N}} \int_{v_{1}} \cdots \int_{v_{n}} \chi^{2} d v_{1} \cdots d v_{n} d s}\right) .
\end{aligned}
$$

Since a $\chi_{k}^{2}$-RF is a collection of a large number of RVs, this results in the evaluation of nested integrals over $B_{r}^{N}$, which is mathematically intractable. In this case, we approximate the intensity measure $\Lambda(\cdot)$ using the expected EC of the $\chi_{k}^{2}-\mathrm{RF}$ excursion set [13].
1) Approximation of Intensity Measure: The expected intensity measure in (14) can be estimated by using topological inference of the average number of upcrossings of the $\chi_{k}^{2}$-RF excursion set above level $u$. This approach is based on the Gaussian kinematic formulae (GKF), given by the following theorem:

Theorem 1. Let $M$ be an $N$-dimensional regular stratified manifold, and $D$ a regular stratified subset of $\mathbb{R}^{k}$. Let $f=$ $\left(f_{1}, \ldots, f_{k}\right): M \rightarrow \mathbb{R}^{k}$ be a vector-valued Gaussian field with i.i.d., components, and $f$ being the Morse function ${ }^{7}$ over $M$ with probability 1 . Then

$$
\mathbb{E}\left\{\mathcal{L}_{i}\left[M \cap f^{-1}(D)\right]\right\}=\sum_{j=0}^{N-i}\left[\begin{array}{c}
i+j \\
j
\end{array}\right] \frac{\mathcal{L}_{i+j}(M) \mathcal{M}_{j}(D)}{(2 \pi)^{\frac{j}{2}}},
$$

where $\mathcal{L}_{i+j}$ for $i=0, \ldots, N, j=0, \ldots, N-i$, are LipschitzKilling curvature measures on $M$ with respect to the metric induced by $f$, and $\mathcal{M}_{j}$ are the generalized (Gaussian) Minkowski functionals on $\mathbb{R}^{k}$. For notational convenience, we define the combinatorial flag coefficients $\left[\begin{array}{c}i+j \\ j\end{array}\right]=\left[\begin{array}{l}a \\ b\end{array}\right]$ as:

$$
\left[\begin{array}{l}
a \\
b
\end{array}\right]=\frac{[a] !}{[b] ![a-b] !}, \quad[a] !=a ! \omega_{a}, \quad \omega_{a}=\frac{\pi^{\frac{a}{2}}}{\Gamma\left(\frac{a}{2}+1\right)} .
$$

Proof. See [13, Theorem 15.9.4]

Using Theorem 1 and (5), the expected intensity measure can be approximated as:

$$
\begin{aligned}
\psi_{0} & \approx \mathbb{E}\left[\chi_{k}^{2}\left(B_{r}^{N}\right)\right], \\
& \approx \mathbb{E}\left\{\mathcal{L}_{0}\left[A_{u}\left(\chi_{k}^{2}, B_{r}^{N}\right)\right]\right\}, \\
& \approx \sum_{j=0}^{N} \frac{\mathcal{L}_{j}\left(B_{r}^{N}\right) \mathcal{M}_{j}(D)}{(2 \pi)^{\frac{j}{2}}} .
\end{aligned}
$$

The Minkowski functionals $\mathcal{M}_{j}(D)$ can be transformed into

\footnotetext{
${ }^{7}$ For the definition of the Morse function and Morse's theorem, refer to [32, Theorem 4.4.1, pp. 87] and [28, Sec. 9.3, Definition 9.3.1, pp. 206].
} 


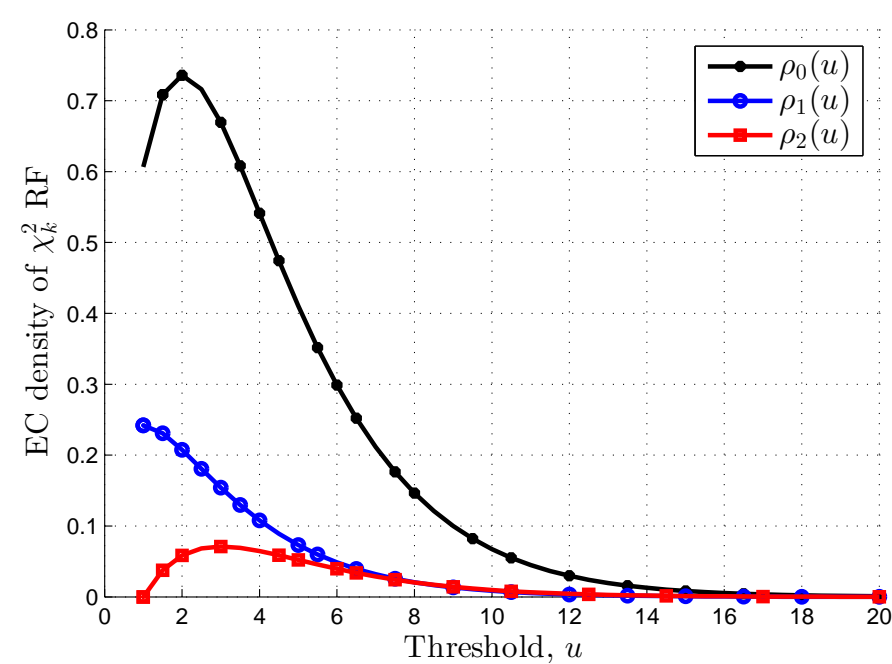

Fig. 4: EC density representing unit-component density of a $\chi_{k}^{2}$-RF that survives threshold $u$.

an EC density for a $\chi_{k}^{2}$-RF as [13]:

$$
\psi_{0} \approx \sum_{j=0}^{N} \rho_{j}(u) \mathcal{L}_{j}\left(B_{r}^{N}\right),
$$

where

$$
\begin{aligned}
\rho_{j}(u)= & \frac{u^{\frac{k-j}{2}} e^{-\frac{u}{2}}}{(2 \pi)^{\frac{j}{2}} \Gamma\left(\frac{k}{2}\right) 2^{\frac{k-2}{2}}} \sum_{l=0}^{\left\lfloor\frac{j-1}{2}\right\rfloor j-1-2 l} \sum_{m=0}^{j-1} \\
& \times \mathbb{1}_{\{k \geq j-m-2 l\}}\left(\begin{array}{c}
k-1 \\
j-1-m-2 l
\end{array}\right) \\
& \times \frac{(-1)^{j-1+m+l}(j-1) !}{m ! l ! 2^{l}} u^{m+l} .
\end{aligned}
$$

The EC density over $\mathbb{R}^{2}$ and average up-crossings of a $\chi_{k}^{2}$ RF are shown in Fig. 4 and Fig. 5, respectively. In Fig. 4, $\rho_{j}(\cdot)$ depicts the unit-component density of a $\chi_{k}^{2}$-RF over the topology of manifold $M$ that survives the threshold $u$, where the value of $j$ defines the dimension of manifold $M$, as discussed in Section II-4. In the case of $j=0$, the topology is transformed from an $N$-dimensional manifold to zero dimension, which is effectively a point. For $j=1$ and $j=2$, the EC density provides the unit-component density of the field over the boundary length and area, respectively. In Fig. 5, the upcrossing behavior of a $\chi_{k}^{2}$-RF for different $u$ is plotted with respect to different $\mathcal{L}_{i}$ measures. In this paper, we consider $\mathcal{L}_{0}$ (i.e., the EC) to approximate the intensity measure of a PCP process.

2) Poisson Clumping Heuristic ${ }^{8}$ : To approximate the nearest-neighbor distribution function, we consider the probability of getting a cluster of potential D2D pairs with spatial distance $r$ or larger above threshold $u$. The general expression for this cluster level inference is given as [14], [34]:

$$
G(r) \approx 1-e^{-\psi_{0} p(v \geq r)} .
$$

\footnotetext{
${ }^{8}$ At high thresholds, the clusters in the excursion set can be regarded as a multidimensional point process with no memory, and hence they behave as Poisson clumps [33].
}

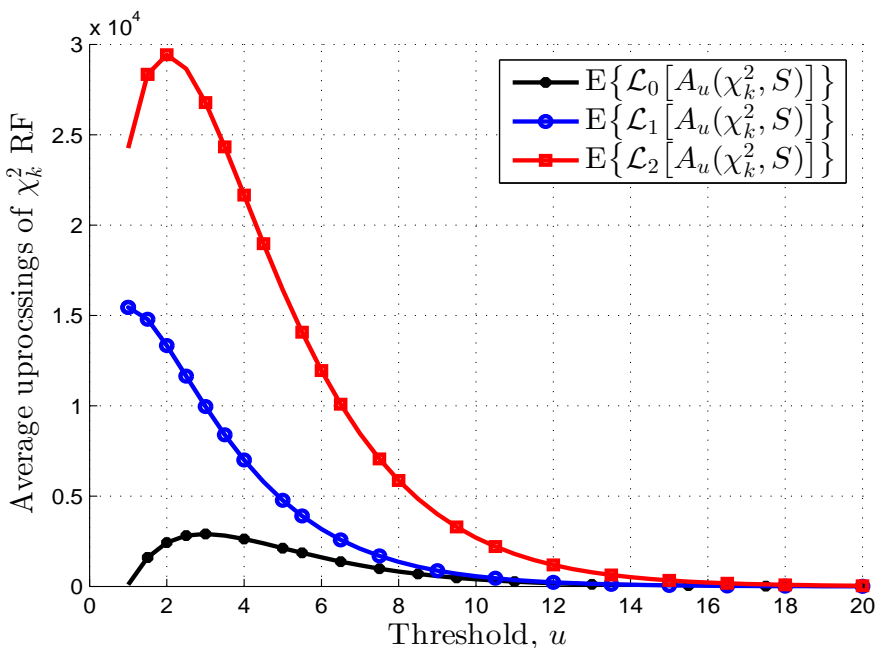

Fig. 5: Average upcrossings of a $\chi_{k}^{2}$-RF for different $\mathcal{L}_{i}$ over a $200 \times 200$ square grid.

The volume $v$ of clusters over spatial distance $r$ is distributed according to [35]:

$$
p(v \geq r) \approx e^{\left(-\beta r \frac{2}{N}\right)},
$$

where $\beta=\left[\frac{\Gamma\left(\frac{N}{2}+1\right)}{\eta}\right]^{\frac{2}{N}}$, and $\eta=\rho_{0}^{n} \mathcal{L}_{N} / \psi_{0}$ is the expected volume of each cluster. The above two equations, (16) and (17), are generalized expressions for $n$ SPMs. In the cellular domain, $n$ SPM means $n$ cellular coverage areas with D2D communication. Since, we are providing the analysis of one coverage area, the order of EC density $\rho_{0}^{n}$ is considered as 1. Plots of $\eta$ and $p(v \geq r)$ are shown in Fig. 6 and Fig. 7, respectively. In these figures, we can see that the maximum expected volume and the probability of having nodes with spatial distance $\geq r$ occurs for $n=1$ which is the first cluster or Poisson clump in a multidimensional point process. In deriving a CFA of the $G$ function, we, consider $\eta$ for $n=1$. The expected volume of cluster for $n=1$ shows all potential D2D nodes that survived the threshold $u$ for a given distance $r$ (see Fig. 9).

Using (6), the Lipschitz-Killing curvature measures over a ball of spatial distance $r$ can be derived as:

$$
\begin{aligned}
& \mathcal{L}_{0}=1, \\
& \mathcal{L}_{1}=2 \sqrt{\pi} r \frac{\Gamma\left(\frac{1}{2}+1\right)}{\Gamma(2)}, \\
& \mathcal{L}_{2}=\pi r^{2} \frac{\Gamma(1)}{\Gamma(2)} .
\end{aligned}
$$

Considering (18), (15), and (17) in (16), the $G$ function can be approximated for, at most, distance $r$. A plot of the $G$ function can be seen in Fig. 8, where the PCP points, due to positive spatial correlation, have higher probability of D2D pairs as compared to SPPP points. For example, at a distance of $2.5 \mathrm{~m}$, the probability of two spatially correlated potential candidates for $\mathrm{D} 2 \mathrm{D}$ communication is 0.8 for $\mathrm{PCP}$ points as compared to 0.42 for SPPP points, which occur so close by chance (i.e., not due to spatial correlation under some homophilic relation). This is because an SPPP cannot 
model spatial correlation between points and is characterized by complete spatial randomness.

\section{Average Coverage Probability}

In this section, we introduce the $G$ function as the retention probability of D2D nodes at spatial distance $r$ to analyze the interference and resulting average coverage probability of a cellular user. We assume an interference-limited environment due to a large number of potential D2D pairs. Hence, the signal-to-interference ratio (SIR) is given as:

$$
\mathrm{SIR}_{\mathrm{SBS}}=\frac{p_{\mathrm{c}} f_{\mathrm{c}} r_{\mathrm{c}}^{-\alpha}}{\sum_{i \in \Phi} p_{\mathrm{i}} f_{\mathrm{i}} r_{\mathrm{i}}^{-\alpha}},
$$

where $p_{\mathrm{c}}$ and $p_{\mathrm{i}}$ are the transmit powers of the cellular user and D2D interferers, respectively, and likewise, $f_{\mathrm{c}}$, and $f_{\mathrm{i}}$ are small-scale fading coefficients. The corresponding distancedependent path losses are $r_{\mathrm{c}}^{-\alpha}$ and $r_{\mathrm{i}}^{-\alpha}$. Assuming an exponential distribution with mean 1 for the power received by the SBS, the average coverage probability of an uplink cellular user is given by the following theorem.

Theorem 2. The average coverage probability of a cellular user with underlay D2D communication is:

$$
p_{\mathrm{cov}}^{\mathrm{c}} \approx \int_{R 0}^{R} e^{-\frac{2 \pi^{2} \psi_{0} p(r) r_{\mathrm{c}}^{2}}{\alpha \sin \left(\frac{2 \pi}{\alpha}\right)}\left(\frac{\gamma}{p_{\mathrm{c}}}\right)^{\frac{2}{\alpha}} \mathbb{E}\left[p_{\mathrm{i}}^{\frac{2}{\alpha}}\right]} \frac{2 r_{\mathrm{c}}}{R^{2}} d r_{\mathrm{c}} .
$$

Proof. See appendix A.

For the same transmit power of all D2D interferers, the average coverage probability of a cellular user for path loss exponent $\alpha=4$ and $R_{0} \approx 0$ reduces to:

$$
p_{\mathrm{cov}}^{\mathrm{c}} \approx \frac{e^{-\frac{\pi^{2} R^{2} \psi_{0} p(r)}{2} \sqrt{\frac{\gamma p_{\mathrm{i}}}{p_{\mathrm{c}}}}}-1}{-\frac{\pi^{2} R^{2} \psi_{0} p(r)}{2} \sqrt{\frac{\gamma p_{\mathrm{i}}}{p_{\mathrm{c}}}}} .
$$

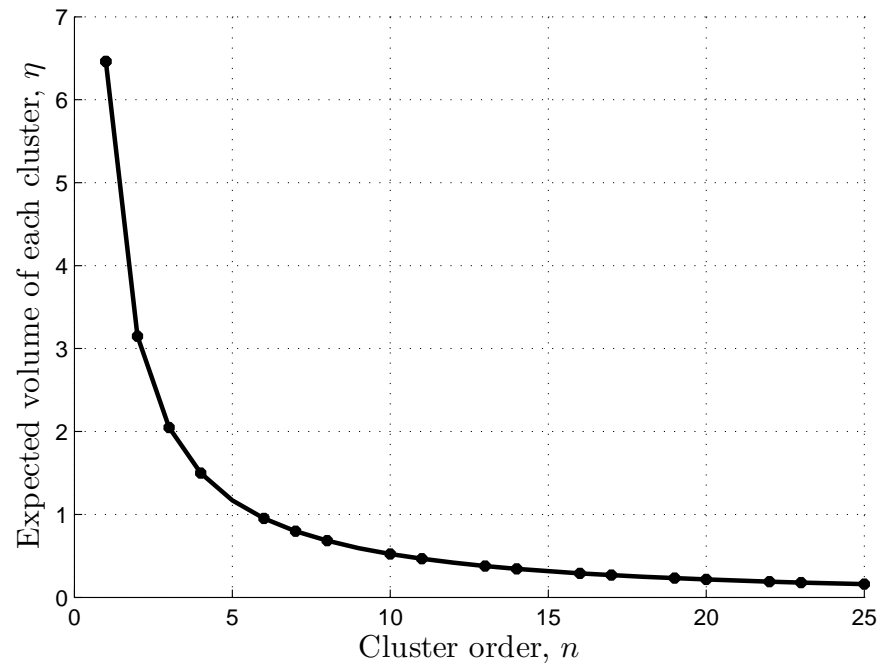

Fig. 6: Expected volume of each cluster for $u=31$, which depends on the EC density, volume of underlying space, and expected EC.

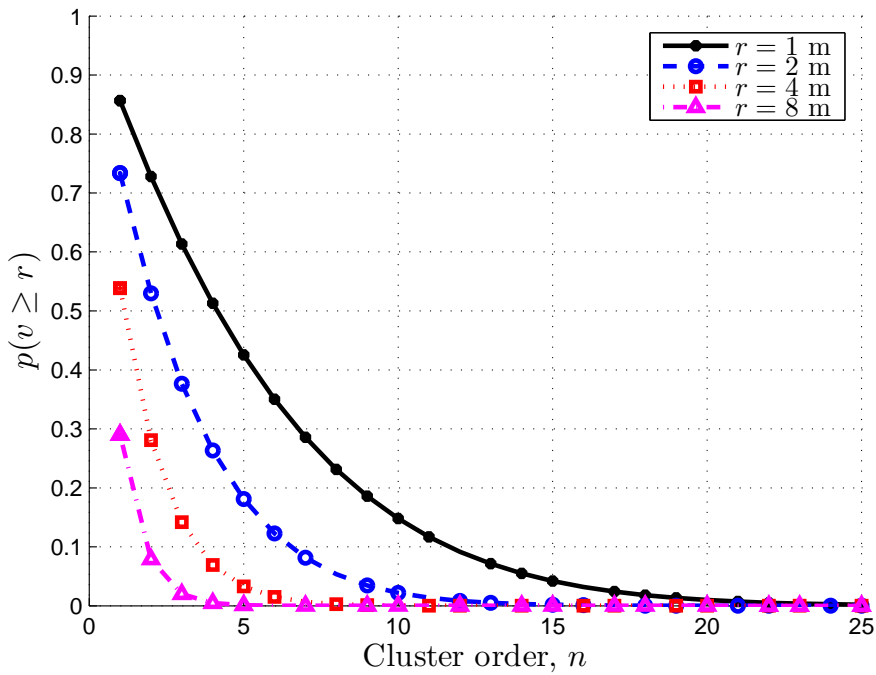

Fig. 7: The probability of cluster number $n$ with different spatial distance $r$, which is exponentially distributed with mean $\beta[$ see (17)].

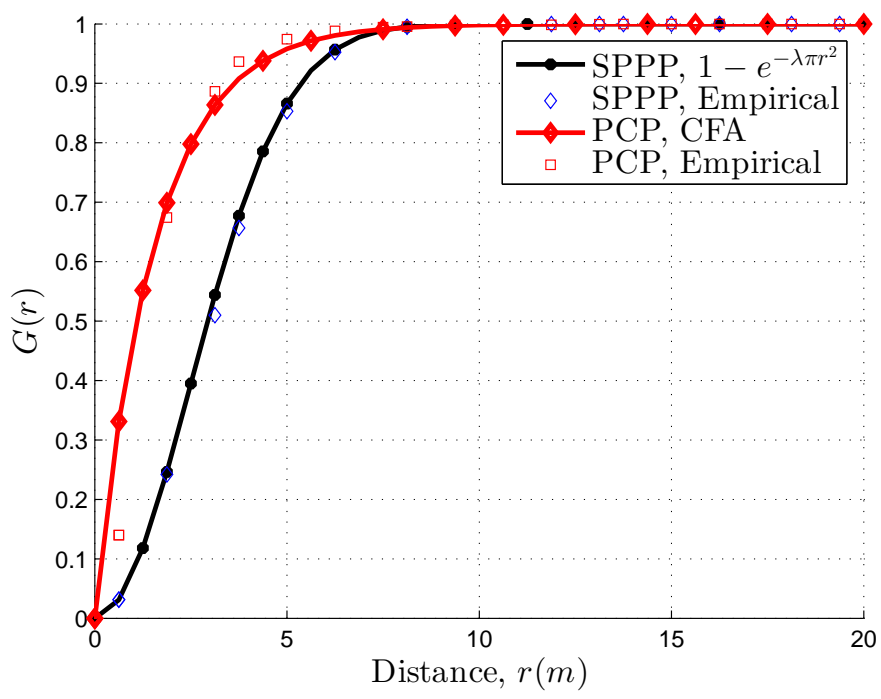

Fig. 8: Nearest-neighbor distribution function of a PCP shows higher probability for lower values of $r$ as compared to a homogeneous SPPP.

\section{NumericAl Results AND Discussion}

In this section, we numerically evaluate the analytic expressions of Section V using Monte-Carlo-based approximations of a PCP process. The average coverage probability of a cellular user (19) depends on $R, \psi_{0}$ (as well as an important implicit parameter, $u$ ), spatial distance $r$, D2D transmit power $p_{\mathrm{i}}$, transmit power of the cellular user $p_{\mathrm{c}}$, and target threshold $\gamma$. The first and foremost step is to identify the implicit parameter $u$, which is introduced as an interference control parameter for D2D pairing. Parameter $u$ is a function of grid size and, more specifically, SBS radius $R$. After finding the feasible range of $u$ for a given radius $R$, we varied the other parameters to analyze the average coverage probability of a cellular user. Then, for different spatial distances $r$, the cumulative interference effect is captured. Since the distance between D2D pairs is much smaller than the distance between the D2D pair and the SBS, it is reasonable to assume the 


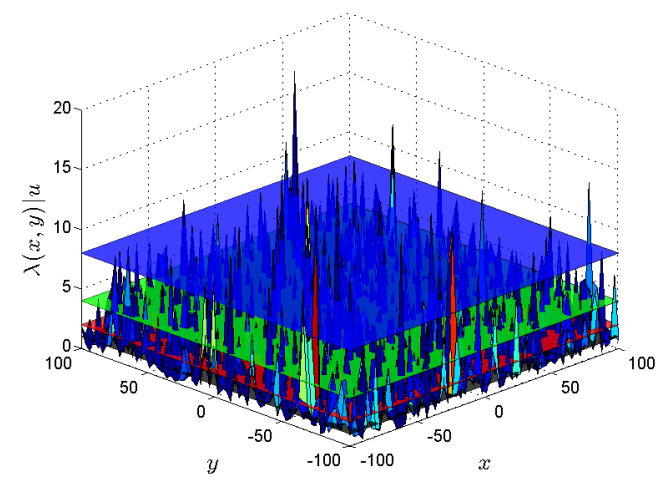

(a)

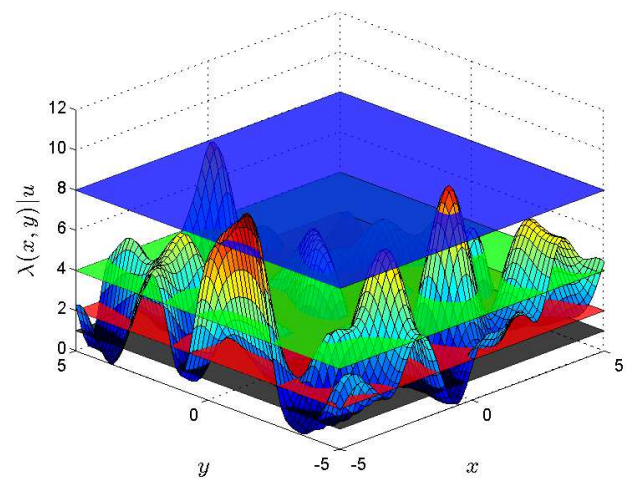

(b)

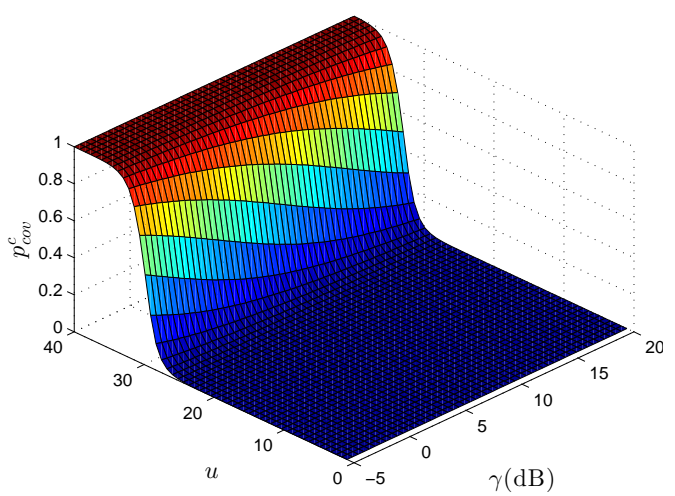

(c)

Fig. 9: Interference characterization for $p_{\mathrm{i}}=0 \mathrm{dBm}, p_{\mathrm{c}}=20 \mathrm{dBm}$, and $R=100 \mathrm{~m}$. (a) Intensity function of a PCP on $200 \times 200$ grid for threshold values $u=(1,2,4,8)$. (b) $10 \times 10$ extract of (a) shows surviving and departing blobs at same values of $u$. (c) Average coverage probability of a cellular user, which drops for low values of $u$, i.e., maximum number of possible D2D pairs.

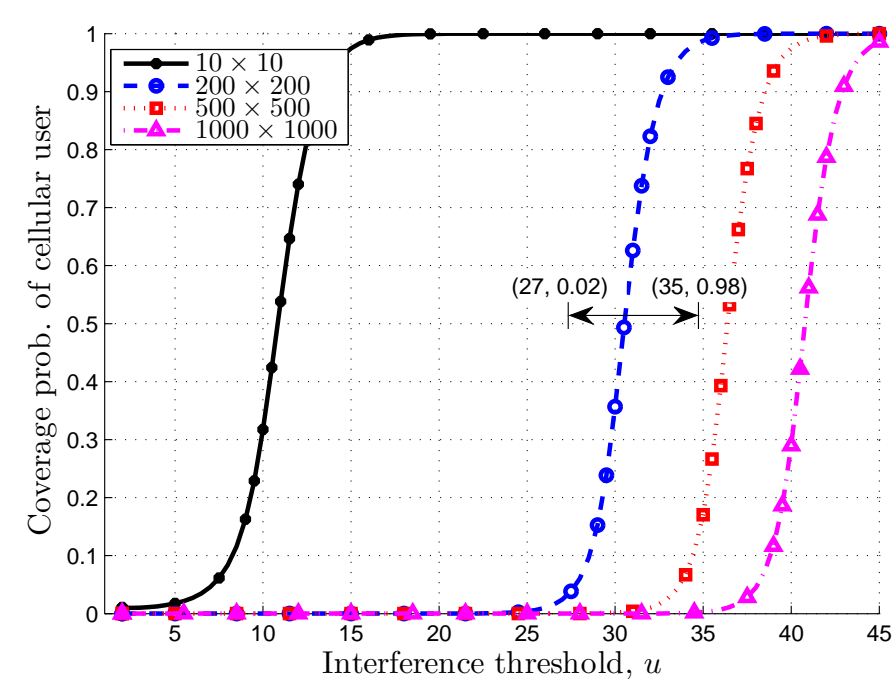

Fig. 10: Effect of interference control parameter $u$ on coverage probability for different grid sizes (SBS radius $R$ ), $p_{\mathrm{i}}=0 \mathrm{dBm}$, and $p_{\mathrm{c}}=20 \mathrm{dBm}$.

same transmit power for every $\mathrm{D} 2 \mathrm{D}$ pair in the coverage area. To analyze the interference due to $\mathrm{D} 2 \mathrm{D}$ clusters, we introduce the nearest-neighbor distribution function into the Laplace functional of the PCP.

In Fig. 9, we show the intensity function at different thresholds and corresponding average coverage probability of a cellular user for grid size $200 \times 200$ (SBS of radius $R=$ $100 \mathrm{~m}$ ). For clear illustration, a small portion of this grid is shown in Fig. 9(b). On this scale, if we set $u=2$ (transparent black plane), all nodes (despite low spatial correlation) will be considered to make D2D pairs ${ }^{9}$ based on the spatial distance $r$. This results in maximum intracell interference and causes blockage for the cellular user. If we increase $u$ (red, green, and blue transparent planes), only those potential D2D pairs that lie under high intensity blobs of the $\chi_{k}^{2}$-RF will survive. In this case, the coverage probability of a cellular user can be ensured while reusing the resources for D2D pairs. The coverage probability curves for different $u$ and $\gamma$ can be seen in Fig. 9(c). A high threshold, for example, $u=35$ in this figure, shows no D2D pair and ensures unit coverage probability of a cellular user.

The interference control parameter $u$ for different grid sizes is plotted in Fig. 10, where it can be seen that the effect of interference on coverage probability due to D2D communication is captured by $u$. As an example, for a $10 \times 10$ grid size (SBS of radius $R=5 \mathrm{~m}$ ), $p_{\mathrm{cov}}^{\mathrm{c}}$ rises from $2 \%$ to $98 \%$ for $u=5$ to 16 , as compared to a $1000 \times 1000$ grid size $(R$ $=500 \mathrm{~m}$ ) where the blockage extends from the floor up to a value of $u=37$, and shows a $98 \%$ rise in $p_{\mathrm{cov}}^{\mathrm{c}}$ at $u=45$.

\footnotetext{
${ }^{9}$ The maximum number of upcrossings of a $\chi_{k}^{2}$-RF occurs at around $u=k$, as can be verified by the plots of Euler density (Fig. 4) and expected EC (Fig. 5).
} 


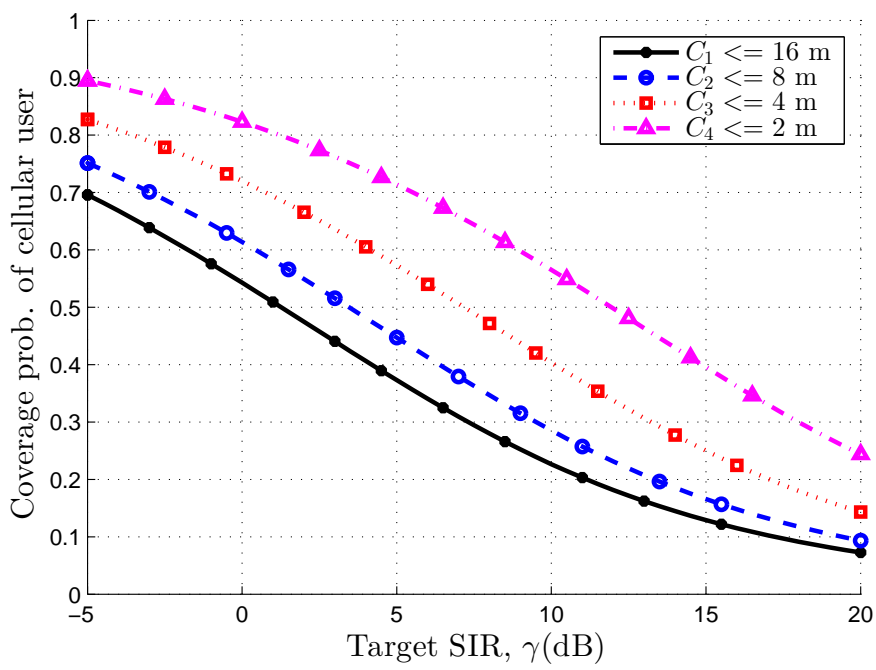

Fig. 11: Effect of interference on coverage probability for different cluster sizes of D2D nodes with $u=31, p_{\mathrm{i}}=0 \mathrm{dBm}, p_{\mathrm{c}}=20 \mathrm{dBm}$, and $R=100$ $\mathrm{m}$.

In Fig. 11, we show the effect of interference due to different cluster sizes on coverage probability. The cluster sizes show different numbers of D2D nodes that survive the threshold $u$. For example, at $u=31\left(p_{\text {cov }}^{\mathrm{c}}=0.62\right.$ from Fig. 10), four cluster sizes of $r=(16,8,4,2) \mathrm{m}$ are shown that consider D2D communication by reusing the frequency of a cellular user. The maximum cluster size considers all D2D nodes that are within $16 \mathrm{~m}$, and hence causes maximum interference. Contrary to this, the minimum cluster size considers nodes within $2 \mathrm{~m}$, and hence results in less interference.

The effect of interference power control on D2D pairs can be seen in Fig. 12, where the coverage probability can be ensured by controlling the transmit power of successful D2D pairs. The coverage drop over the range of $\gamma$ between 0 and $20 \mathrm{~dB}$ is approximately equal. The two upper curves (lower $p_{\mathrm{i}}$, i.e., $-20 \mathrm{dBm}$ and $\left.-10 \mathrm{dBm}\right)$ at lower target SIR, e.g., $\gamma=$ $-5 \mathrm{~dB}$, are closer to each other, whereas the same curves are apart from each other at higher target SIR, e.g., $\gamma=20 \mathrm{~dB}$. This trend is reversed for the two lower curves.

The effect of user power control on coverage probability is shown in Fig. 13. It can be seen that the curves for different $p_{\mathrm{c}}$ converge to low coverage probability for high $\gamma$. The coverage probability can be increased by either reducing the transmit power of D2D pairs or reducing the number of D2D pairs by increasing the threshold $u$. The threshold $u$ and spatial distance $r$ (small $r$ requires lower $p_{\mathrm{i}}$ ) are key control parameters to ensure the extent of frequency reuse (D2D pairs) while ensuring coverage probability of a cellular user.

\section{CONCLUSION}

In this paper, we considered inhomogeneity, positive spatial correlation, and random intensity in the spatial modeling of MTs in the coverage area. In conventional SPPP-based modeling, these assumptions are relaxed and hence provides overly simplistic spatial modeling of MTs. For precise modeling, we assumed a PCP process to capture inhomogeneous and

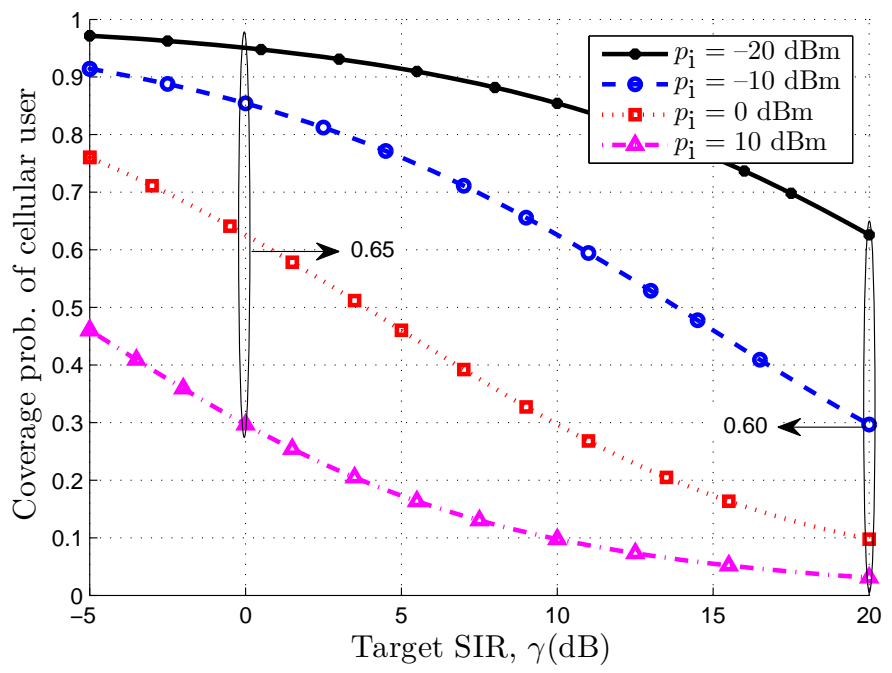

Fig. 12: Effect of interference power control on coverage probability for D2D pairs with $u=31, p_{\mathrm{c}}=20 \mathrm{dBm}$, and $R=100 \mathrm{~m}$.

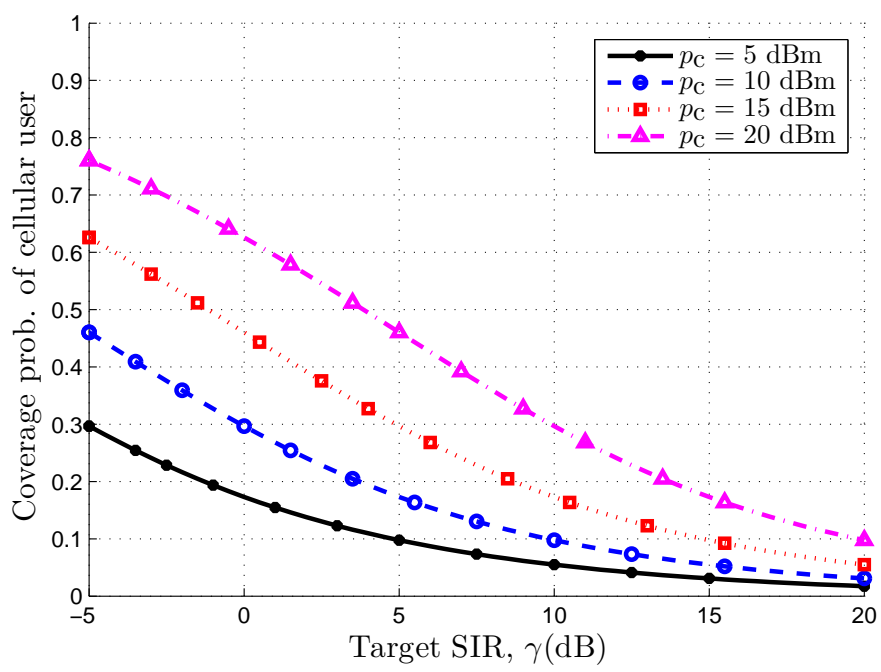

Fig. 13: Effect of user power control on coverage probability for D2D pair with $u=31, p_{\mathrm{i}}=0 \mathrm{dBm}$, and $R=100 \mathrm{~m}$.

spatially correlated MTs. We considered this process to characterize intracell interference in a D2D underlay network. We further approximated the intractable nearest-neighbor distribution function by adopting the expected Euler Characteristic and Poisson clumping heuristic. The key findings of this research are enumerated as:

1) Since a simple SPPP process with constant intensity measure cannot capture the prevailing inhomogeneity and spatial correlation in dense cellular networks, point processes with an attraction/repulsion property (e.g., Cox process/DPP) are potential candidates for precise spatial modeling of MTs/BSs.

2) The Euler Characteristic and RFT framework can be used to analyze and identify high-intensity areas/hotspots for D2D communication.

3) If statistical parameter maps of the coverage area are available, statistical inference can be performed to identify clusters of MTs with high spatial correlation (po- 
tential areas for D2D communication).

4) The intensity measure of a PCP is governed by a $\chi_{k}^{2}-\mathrm{RF}$. In this case, the threshold $u$ of the excursion set plays a key role in D2D communication to control the cluster size, the level of interference due to frequency reuse, and the coverage probability of a cellular user.

\section{ACKNOWLEDGEMENTS}

This research was partly funded by EPSRC Global Challenges Research Fund - the DARE project - EP/P028764/1. The statements made herein are solely the responsibility of the authors. We would like to acknowledge the support of the University of Surrey 5GIC (http://www.surrey.ac.uk/5gic) members for this work.

\section{APPENDIX A - PROOF OF THEOREM 4}

The average coverage probability of an uplink cellular user distributed uniformly over a plane between $R$ and $R_{0}$ at a distance $r_{\mathrm{c}}$ from the serving SBS is given as:

$$
\begin{aligned}
p_{\mathrm{cov}}^{\mathrm{c}} & =\mathbb{E}_{r_{\mathrm{c}}}\left[p\left(\operatorname{SIR}_{\mathrm{SBS}} \geq \gamma \mid r_{\mathrm{c}}\right)\right], \\
& =\mathbb{E}_{r_{\mathrm{c}}}\left[p\left(f_{\mathrm{c}} \geq \frac{\gamma I_{\mathrm{m}}}{p_{\mathrm{c}} r_{\mathrm{c}}^{-\alpha}} \mid r_{\mathrm{c}}\right)\right],
\end{aligned}
$$

where

$$
I_{\mathrm{m}}=\sum_{i \in \Phi} p_{\mathrm{i}} f_{\mathrm{i}} r_{\mathrm{i}}^{-\alpha}
$$

is the cumulative interference due to D2D clusters in the coverage area and $\mathbb{E}_{(\cdot)}$ is expectation with respect to $(\cdot)$.

In (A.1), the coverage probability depends on a number of RVs, i.e., $p_{\mathrm{c}}, f_{\mathrm{c}}, r_{\mathrm{c}}^{-\alpha}, p_{\mathrm{i}}, f_{\mathrm{i}}, r_{\mathrm{i}}^{-\alpha}$. The power transmitted by the cellular user $p_{\mathrm{c}}$ is assumed to be independent of the interferers. The serving SBS uses uplink power control to ensure the quality of service for the cellular user, based on a distancedependent path loss. The fading coefficients $f_{\mathrm{c}}$ and $f_{\mathrm{i}}$ follow a Rayleigh distribution with $p_{\mathrm{c}}$ and $p_{\mathrm{i}}$ exponentially distributed. The cellular user is uniformly distributed in the coverage area, whereas all potential D2D nodes are distributed according to a PCP process. Conditioning on $g=\left\{p_{\mathrm{i}}, f_{\mathrm{i}}\right\}$, the coverage probability of a cellular user for a given transmit power $p_{\mathrm{c}}$ is:

$$
\begin{aligned}
p\left(\operatorname{SIR}_{\mathrm{SBS}} \geq \gamma \mid r_{\mathrm{c}}, g\right) & =\int_{x=\frac{\gamma I_{\mathrm{m}}}{p_{\mathrm{c}} r_{\mathrm{c}}^{-\alpha}}}^{\infty} e^{-x} d x \\
& =e^{-\gamma p_{\mathrm{c}}^{-1} r_{\mathrm{c}}^{\alpha} I_{\mathrm{m}}} .
\end{aligned}
$$

De-conditioning by $g$, (A.3) results in:

$$
\begin{aligned}
p\left(\operatorname{SIR}_{\mathrm{SBS}} \geq \gamma \mid r_{\mathrm{c}}\right) & =\mathbb{E}_{g}\left(e^{-\gamma p_{\mathrm{c}}^{-1} r_{\mathrm{c}}^{\alpha} I_{\mathrm{m}}}\right), \\
& =\mathbb{E}_{g}\left(e^{-s_{\mathrm{c}} I_{\mathrm{m}}}\right), \\
& =\mathcal{L}_{I_{\mathrm{m}}}\left(s_{\mathrm{c}}\right)
\end{aligned}
$$

where $s_{\mathrm{c}}=\gamma p_{\mathrm{c}}^{-1} r_{\mathrm{c}}^{\alpha}$.
Putting the value of $I_{\mathrm{m}}$ from (A.2) in (A.4)

$$
\begin{aligned}
\mathcal{L}_{I_{\mathrm{m}}}\left(s_{\mathrm{c}}\right) & =\mathbb{E}_{\Phi, p_{\mathrm{i}}, f_{\mathrm{i}}}\left(e^{-s_{\mathrm{c}} \sum_{i \in \Phi} p_{\mathrm{i}} f_{\mathrm{i}} r_{\mathrm{i}}^{-\alpha}}\right) \\
& =\mathbb{E}_{\Phi, p_{\mathrm{i}}, f_{\mathrm{i}}}\left(\prod_{i \in \Phi} e^{-s_{\mathrm{c}} p_{\mathrm{i}} f_{\mathrm{i}} r_{\mathrm{i}}^{-\alpha}}\right) \\
& =\mathbb{E}_{\Phi}\left[\prod_{i \in \Phi} \mathbb{E}_{p_{\mathrm{i}}}\left(\frac{1}{1+s_{\mathrm{c}} p_{\mathrm{i}} r_{\mathrm{i}}^{-\alpha}}\right)\right] \\
& \approx \mathbb{E}_{\Phi}[\prod_{i \in \Phi} \underbrace{\left(\frac{1}{1+s_{\mathrm{c}} \mathbb{E}\left[p_{\mathrm{i}}\right] r_{\mathrm{i}}^{-\alpha}}\right)}_{f(x)}]
\end{aligned}
$$

where (A.5) results from the i.i.d. assumption of $p_{\mathrm{i}}$ and $f_{\mathrm{i}}$, and further independence from the PCP process. The expected value is a linear operator and the value of $p_{\mathrm{i}}$ is usually varied in discrete steps within a small range, e.g., $[-5,-4, \cdots, 5]$ $\mathrm{dBm}$. In this case, $\mathbb{E}_{p_{\mathrm{i}}}(\cdot)$ in $(\mathrm{A} .5)$ can be approximated by $\mathbb{E}\left[p_{\mathrm{i}}\right]$.

The Laplace functional ${ }^{10}$ of a general Cox process is given as [37]:

$$
\mathcal{L}_{\Phi}(f)=\mathbb{E}_{\Lambda}\left[e^{-\int_{\mathbb{R}^{2}}[1-f(x)] \Lambda(x) d x}\right]
$$

In (A.6), the random intensity measure $\Lambda(x)$ defines the Laplace functionals for subclasses of a Cox process. For example, the same Laplace functional ${ }^{11}$ holds for a PCP process if $\Lambda(x)$ is defined as in (3). The expression in (A.6) is mathematically intractable since the integral over $\mathbb{R}^{2}$ for nested integrals of $\Lambda(x)$ (governed by a $\chi_{k}^{2}$-RF) can not be solved in closed-form, as discussed in Section IV below (14). Therefore, we apply the approximations of Theorem 3 and replace $\Lambda(x)$ in (A.6) by $\psi_{0}$, i.e., the expected intensity. In this case, the outer expectation operator can be ignored since $\psi_{0}$ is already an average value of $\Lambda(x)$. The Laplace functional of a PCP process, in this case, can be given as:

$$
\begin{aligned}
\mathbb{E}_{\Phi}\left[\prod_{i \in \Phi} f(x)\right] & \approx e^{-\int_{\mathbb{R}^{2}}[1-f(x)] \psi_{0} d x} \\
& \stackrel{(a)}{\approx} e^{-\int_{\mathbb{R}^{2}}[1-f(x)] p(r) \psi_{0} d x} \\
& \approx e^{-\psi_{0} p(r) \int_{0}^{\infty} \int_{0}^{2 \pi}[1-f(x)] x d \theta d x} \\
& \approx e^{-2 \pi \psi_{0} p(r) \int_{0}^{\infty}[1-f(x)] x d x}
\end{aligned}
$$

where $(a)$ results by applying thinning [36, Proposition 1.3.5] based on the retention probability $p(r)=G(r)$ from (16).

\footnotetext{
${ }^{10}$ The Laplace functionals of a general point process and a PPP are, respectively, given in [36, Definition 1.2.1 and Proposition 1.2.2].

${ }^{11}$ For a GRF $X$, PPP $\Phi$, kernel function $h(\cdot, \cdot)$, Lévy basis $L(\cdot)$, and random parameter $\lambda^{*}>0$, the random intensity function $\lambda(s)$ for different subclasses of Cox processes are given as:

- Log Gaussian: $\lambda(s)=\exp [X(s)]$.

- Generalized Shot Noise: $\lambda(s)=\sum_{X \in \Phi} h(X, s)$.

- Lévy Based: $\lambda(s)=\int h(x, s) L(d x)$.

- Permanental: $\lambda(s)=X_{1}^{2}(s)+\cdots+X_{k}^{2}(s)$.

- Sigmoidal Gaussian: $\lambda(s)=\lambda^{*} /\{1+\exp [-X(s)]\}$.

The random intensity function can be plugged into (3) to find the random
} intensity measure $\Lambda(s)$ for the respective subclasses of the Cox process. 
Putting $f(x)$ from (A.5) in (A.7), $\mathcal{L}_{I_{\mathrm{m}}}(\cdot)$ can be computed as:

$$
\begin{aligned}
\mathcal{L}_{I_{\mathrm{m}}}\left(s_{\mathrm{c}}\right) & \approx e^{-2 \pi \psi_{0} p(r) \int_{R_{0}}^{\infty}\left(1-\frac{1}{1+s_{\mathrm{c}} \mathbb{E}\left[p_{\mathrm{p}}\right] x^{-\alpha}}\right) x d x}, \\
& \approx e^{-2 \pi \psi_{0} p(r) \int_{R_{0}}^{\infty}\left(\frac{1}{1+\frac{x^{\alpha}}{s_{\mathrm{c}} \mathbb{E}\left[p_{\mathrm{i}}\right]}}\right) x d x} .
\end{aligned}
$$

By substituting $\frac{x^{\alpha}}{s_{\mathrm{c}} \mathbb{E}\left[p_{\mathrm{i}}\right]}=u^{\alpha}$, (A.8) can finally be expressed as:

$$
\mathcal{L}_{I_{\mathrm{m}}}\left(s_{\mathrm{c}}\right) \approx e^{-2 \pi \psi_{0} p(r)\left(s_{\mathrm{c}}\right) \frac{2}{\alpha} \mathbb{E}\left[p_{\mathrm{i}}^{\frac{2}{\alpha}}\right] \int_{R_{0}}^{\infty}\left(\frac{u}{1+u^{\alpha}}\right) d u} .
$$

Since $R_{0} \ll R$, and assuming $R_{0} \approx 0$, the integral on the right hand side of (A.9) can be evaluated as:

$$
\int_{0}^{\infty}\left(\frac{u}{1+u^{\alpha}}\right) d u=\frac{\pi}{\alpha \sin \left(\frac{2 \pi}{\alpha}\right)}
$$

Putting (A.10) in (A.9) and using the uniform distribution from (7), the average coverage probability of a cellular user (A.1) is finally expressed as:

$$
\begin{aligned}
p_{\mathrm{cov}}^{\mathrm{c}} & \approx \mathbb{E}_{r_{\mathrm{c}}}\left[e^{-\frac{2 \pi^{2} \psi_{0} p(r) r_{\mathrm{c}}^{2}}{\alpha \sin \left(\frac{2 \pi}{\alpha}\right)}\left(\frac{\gamma}{p_{\mathrm{c}}}\right)^{\frac{2}{\alpha}} \mathbb{E}\left[p_{\mathrm{i}}^{\frac{2}{\alpha}}\right]} \mid r_{\mathrm{c}}\right], \\
& \approx \int_{R 0}^{R} e^{-\frac{2 \pi^{2} \psi_{0} p(r) r_{\mathrm{c}}^{2}}{\alpha \sin \left(\frac{2 \pi}{\alpha}\right)}\left(\frac{\gamma}{p_{\mathrm{c}}}\right)^{\frac{2}{\alpha}} \mathbb{E}\left[p_{\mathrm{i}}^{\frac{2}{\alpha}}\right]} \frac{2 r_{\mathrm{c}}}{R^{2}} d r_{\mathrm{c}} .
\end{aligned}
$$

\section{REFERENCES}

[1] R. Heath, M. Kountouris, and T. Bai, "Modeling heterogeneous network interference using Poisson point processes," IEEE Trans. Signal Process., vol. 61, no. 16, pp. 4114-4126, Aug. 2013.

[2] H. Dhillon, R. Ganti, F. Baccelli, and J. G. Andrews, "Modeling and analysis of K-tier downlink heterogeneous cellular networks," IEEE $J$. Sel. Areas Commun., vol. 30, no. 3, pp. 550-560, Apr. 2012.

[3] C.-H. Lee, C.-Y. Shih, and Y.-S. Chen, "Stochastic geometry based models for modeling cellular networks in urban areas," Wireless Networks, vol. 19, no. 6, pp. 1063-1072, Oct. 2012.

[4] www.statista.com, "Number of social media users worldwide 2010-2020 statistic," The Statistics Portal, Accessed: 201801-03. [Online]. Available: http://www.statista.com/statistics/278414/ number-of-worldwide-social-network-users/

[5] T. Shirai and Y. Takahashi, "Random point fields associated with certain Fredholm determinants I: fermion, Poisson, and boson point processes," J. Functional Anal., vol. 205, no. 2, pp. 414 - 463, Dec. 2003.

[6] J. Hough, M. Krishnapur, Y. Peres, and B. Virag, "Determinantal processes and independence," Prob. Surveys, vol. 3, pp. 206-229, 2006.

[7] Y. Li, F. Baccelli, H. Dhillon, and J. G. Andrews, "Statistical modeling and probabilistic analysis of cellular networks with determinantal point processes," IEEE Trans. Commun., vol. 63, no. 9, pp. 3405-3422, Sep. 2015.

[8] N. Deng, W. Zhou, and M. Haenggi, "The Ginibre point process as a model for wireless networks with repulsion," IEEE Trans. Wireless Commun., vol. 14, no. 1, pp. 107-121, Jan. 2015.

[9] Y. Li, F. Baccelli, H. S. Dhillon, and J. G. Andrews, "Fitting determinantal point processes to macro base station deployments," in Proc. Intl. Conf. Global Communs., (GLOBECOM), Dec 2014, pp. 3641-3646.

[10] H. A. Mustafa, M. Z. Shakir, M. A. Imran, A. Imran, and R. Tafazolli, "Coverage gain and device-to-device user density: stochastic geometry modeling and analysis," IEEE Commun. Lett., vol. 19, no. 10, pp. 17421745, Oct. 2015.
[11] H. A. Mustafa, M. Z. Shakir, M. A. Imran, and R. Tafazolli, "Distance based cooperation region for D2D pair," in Proc. Vehicular Technology Conf., (VTC), May 2015, pp. 1-6.

[12] F. Lavancier, J. Møller, and E. Rubak, "Determinantal point process models and statistical inference," J. Roy. Statist. Soc. B, vol. 77, no. 4, pp. 853-877, Sep. 2015.

[13] R. J. Adler and J. E. Taylor, Random Fields and Geometry. New York, USA: Springer-Verlag, 2007.

[14] K. Friston, Statistical Parametric Mapping: The Analysis of Functional Brain Images. London, UK: Academic Press, 2007.

[15] P. Abrahamsen and N. Regnesentral, "A Review of Gaussian Random Fields and Correlation Functions," Norsk Regnesentral, Norwegian Computing Center, Oslo, Norway, Tech. Rep. 917, 1997.

[16] K. J. Worsley, "Local maxima and the expected Euler characteristic of excursion sets of $\chi^{2}, \mathrm{~F}$ and t fields," Adv. Appl. Prob., vol. 26, no. 1, pp. 13-42, 1994.

[17] J. Møller, A. R. Syversveen, and R. P. Waagepetersen, "Log Gaussian Cox processes," Scandinavian J. Stat., vol. 25, no. 3, pp. 451-482, 1998.

[18] R. J. Adler and A. M. Hasofer, "Level crossings for random fields," Ann. Prob., vol. 4, no. 1, pp. 1-12, Feb. 1976.

[19] R. J. Adler, "Excursions above a fixed level by N-dimensional random fields," J. Appl. Prob., vol. 13, no. 2, pp. 276-289, 1976.

[20] P. A. W. Lewis and G. S. Shedler, "Simulation of nonhomogeneous Poisson processes by thinning," Naval Res. Logis. Quarterly, vol. 26, no. 3, pp. 403-413, 1979. [Online]. Available: http: //dx.doi.org/10.1002/nav.3800260304

[21] P. McCullagh and J. Møller, "The permanental process," Adv. Appl. Prob., vol. 38, no. 4, pp. 873-888, Dec. 2006.

[22] S. M. Ross, Introduction to Probability and Statistics for Engineers and Scientists. Boston, USA: Academic Press, 2009.

[23] C. E. Rasmussen and C. K. I. Williams, Gaussian Processes for Machine Learning. Cambridge, USA: MIT Press, 2006.

[24] L. Valiant, "The complexity of computing the permanent," Theoretical Comput. Sci., vol. 8, no. 2, pp. 189-201, 1979. [Online]. Available: http://www.sciencedirect.com/science/article/pii/0304397579900446

[25] S. C. Kou and P. McCullagh, "Approximating the $\alpha$-permanent," Biometrika, vol. 96, no. 3, pp. 635-644, Sep. 2009. [Online]. Available: http://biomet.oxfordjournals.org/content/96/3/635

[26] O. Macchi, "The coincidence approach to stochastic point processes," Adv. Appl. Prob., vol. 7, no. 1, pp. 83-122, 1975.

[27] E. Spodarev, Stochastic Geometry, Spatial Statistics and Random Fields: Asymptotic Methods. Heidelberg, Germany: Springer-Verlag, 2013.

[28] W. L. Martinez and A. R. Martinez, Computational Statistics Handbook with Matlab. Boca Raton, USA: CRC Press, 2007.

[29] B. D. Ripley, "The second-order analysis of stationary point processes," J. Appl. Prob., vol. 13, no. 2, pp. 255-266, Jun. 1976.

[30] J. Møller and P. McCullagh, "The Permanent Process," Aalborg University, Department of Mathematical Sciences, Aalborg, Denmark, Tech. Rep. R-2005-29, 2005.

[31] J. Cao, "The size of the connected components of excursion sets of $\chi^{2}$, t and F fields," Adv. Appl. Prob., vol. 31, no. 3, pp. 579-595, 1999.

[32] R. J. Adler, The Geometry of Random Fields. Philadelphia, USA: Society for Industrial and Applied Mathematics, 2009.

[33] D. Aldous, Probability Approximations via the Poisson Clumping Heuristic. New York, USA: Springer-Verlag, Mar. 2013.

[34] J. C. Mazziotta, A. W. Toga, and R. S. J. Frackowiak, Brain Mapping: The Disorders. San Diego, USA: Academic Press, 2000.

[35] K. J. Friston, K. J. Worsley, R. S. J. Frackowiak, J. C. Mazziotta, and A. C. Evans, "Assessing the significance of focal activations using their spatial extent," Human Brain Mapping, vol. 1, no. 3, pp. 210-220, Jan. 1994.

[36] F. Baccelli and B. Błaszczyszyn, "Stochastic geometry and wireless networks: Volume I Theory," Foundations and Trends in Networking, vol. 3, no. 34, pp. 249-449, 2008.

[37] A. E. Gelfand, P. Diggle, P. Guttorp, and M. Fuentes, Handbook of Spatial Statistics. Boca Raton, USA: CRC Press, 2010. 